\title{
A Cross-Cultural Investigation of the Role of Foot Size in Physical Attractiveness
}

\author{
Daniel M. T. Fessler, Ph.D., ${ }^{11}$ Daniel Nettle, Ph.D., ${ }^{2}$ Yalda Afshar, B.A., ${ }^{3}$ \\ Isadora de Andrade Pinheiro, B.A., ${ }^{4}$ Alexander Bolyanatz, Ph.D., ${ }^{5}$ \\ Monique Borgerhoff Mulder, Ph.D., ${ }^{6}$ Mark Cravalho, Ph.D., ${ }^{4}$ \\ Tiara Delgado, B.A., ${ }^{7}$ Bozena Gruzd, M.A., ${ }^{8}$ Melissa Oliveira Correia, B.A., 4 \\ Daria Khaltourina, M.A., ${ }^{9}$ Andrey Korotayev, Ph.D., ${ }^{9}$ Jocelyn Marrow, M.A., 10 \\ Lucineide Santiago de Souza, B.A., ${ }^{4}$ and Asta Zbarauskaite, M.A. ${ }^{8}$
}

Received January 29, 2004; revision received May 5, 2004; accepted June 2, 2004

\begin{abstract}
Disparate cultural practices suggest that small foot size may contribute to female attractiveness. Two hypotheses potentially explain such a pattern. Sexual dimorphism in foot size may lead observers to view small feet as feminine and large feet as masculine. Alternately, because small female feet index both youth and nulliparity, evolution may have favored a male preference for this attribute in order to maximize returns on male reproductive investment. Whereas the observational hypothesis predicts symmetrical polarizing preferences, with small feet being preferred in women and large feet being preferred in men, the evolutionary hypothesis predicts asymmetrical preferences, with the average phenotype being preferred in men. Using line drawings that varied only in regard to relative foot size, we examined judgments of attractiveness in nine cultures. Small foot size was generally preferred for females, while average foot size was preferred for males. These results provide preliminary support for the hypothesis that humans possess an evolved preference for small feet in females.
\end{abstract}

KEY WORDS: foot size; physical attractiveness; sexual selection; sexual dimorphism.

\section{INTRODUCTION}

Practices such as the frequent wearing of excessively small shoes by U.S. women (Frey, Thompson, Smith, Sanders, \& Horstman, 1993) and female foot binding

${ }^{1}$ Department of Anthropology, University of California, Los Angeles, California.

${ }^{2}$ Division of Psychology, Brain and Behaviour, University of Newcastle, Newcastle upon Tyne, England.

${ }^{3}$ Independent scholar, Berkeley, California.

${ }^{4}$ Department of Sociology, Universidade Federal da Bahia, Salvador, Brazil.

${ }^{5}$ Core Program, Benedictine University, Lisle, Illinois.

${ }^{6}$ Department of Anthropology, University of California, Davis, California.

${ }^{7}$ Independent documentary filmmaker, New York, New York.

${ }^{8}$ Independent scholar, Vilnius, Lithuania.

${ }^{9}$ School of History, Political Science and Law, Russian State University for the Humanities, Moscow, Russia. in historical China (Jackson, 2000) suggest that small feet may be a component of female attractiveness in many cultures. In this study, we present two competing hypotheses to explain an attraction to small female feet. We then test these hypotheses in an investigation of the role of foot size in judgments of female and male attractiveness in nine cultures.

\section{Possible Origins of Common Sex-Specific Aesthetic Preferences}

Attributes such as symmetry (Grammer \& Thornhill, 1994) and clear skin (Fink, Grammer, \& Thornhill, 2001;

\footnotetext{
${ }^{10}$ Committee on Human Development, University of Chicago, Chicago, Illinois.

${ }^{11}$ To whom correspondence should be addressed at Department of Anthropology, University of California, Los Angeles, Los Angeles, California 90095-1553; e-mail: dfessler@anthro.ucla.edu.
} 
see also Curtis, Aunger, \& Rabie, 2004) are considered attractive features of both men and women in many, perhaps all, cultures. While these preferences are deserving of study, of particular interest for the present purposes are sex-specific aesthetic criteria. Candidate sex-specific attributes proposed to date include lighter female skin tone (Van den Berge \& Frost, 1986), neotenous female cephalofacial morphology (Jones, 1995, 1996), greater male muscularity (Frederick \& Haselton, 2004; Dixson, Halliwell, East, Wignarajah, \& Anderson, 2003), greater male height (Nettle, 2002), and sex-specific patterns of fat deposition (Singh, 1993). As discussed below, for any given attribute, two distinct types of explanation address the origins of the corresponding aesthetic preference.

One class of explanations posits that a given attribute may exist because of functional or phylogenetic reasons unconnected to the ability to attract members of the opposite sex; the aesthetic preference for sex-specific versions of the attribute may thus simply reflect people's observations regarding the differences between women and men. For example, the level of skin pigmentation favored by natural selection is a compromise between the need to protect against ultraviolet radiation and the need to produce vitamin $\mathrm{D}$ through exposure to sunlight. Due to the crucial role of vitamin D in pregnancy and lactation, the latter factor looms larger for women than for men, and, in all populations, natural selection has favored lighter skin tone in women (Jablonski \& Chaplin, 2000). The aesthetic preference for women with skin somewhat lighter than the local average, a pattern found in disparate cultures (Van den Berge \& Frost, 1986), may thus derive from a process in which people the world over observe that women tend to be lighter-skinned than men, whereafter lighter skin comes to be associated with femininity, and thus with female attractiveness.

A second class of explanations of aesthetic preferences posits that, rather than stemming from observations regarding evolved differences between female and male morphology, some preferences may themselves be the product of evolution. Substantial evidence supports the claim that men prefer women who exhibit morphological traits associated with youth (Buss, 1994; Symons, 1995). This is particularly striking given that, in many primate species, males disfavor youthful, nulliparous females, a pattern explicable in terms of the latter's low fertility and high miscarriage rates (Manson, 1997), limitations that also characterize youthful, nulliparous women (Kaar \& Jokela, 1998). The human reversal of prevailing male primate mate selection preferences presumably derives from the fact that, unlike many other male primates, men often invest heavily in their mates and offspring, and do so for prolonged periods. A preference for females exhibiting traits associated with youth and nulliparity would have offered high-investing ancestral men two advantages (Buss, 1994; Jones, 1995, 1996; Symons, 1979, 1995). First, men who preferred women who exhibited such traits would have tended to initiate investment when the recipient was at the beginning of her reproductive career. Because men are capable of providing provisions and protection for many decades, preferring to invest in such women would have generally increased the fitness returns on a man's investment, as investing in a woman from the outset of her reproductive career would have maximized the number of children that a man could father by her. Second, because provisioning and protecting children fathered by other men would have generally constituted a fitness-reducing misapplication of investment, by being most attracted to women who exhibited signs of youth and nulliparity, ancestral men would have reduced the likelihood that the recipients of their investment would either already be pregnant with, or already have given birth to, another man's child.

Over evolutionary time, mate selection preferences can exercise a strong selective effect on the sex under scrutiny, as individuals who exhibit the preferred attributes are able to secure the best partners available, thus reaping attendant benefits such as favorable genes for their offspring and high levels of investment from their privileged partners. Under these circumstances, attributes for which natural selection initially favored a preference due to their indexical value can come to be partially dissociated from the traits thus indexed. In humans, this pattern is particularly evident with regard to female cephalofacial morphology. Both hard- and soft-tissue components of the face change with age. Because ancestral men consistently preferred women who appeared young, ancestral women who possessed facial features that gave the appearance of youth experienced greater reproductive success than did women whose features accurately reflected their age; iterated over many generations, this resulted in a neotenization of female facial morphology (i.e., women possess smaller ears and noses than men, the forehead constitutes a greater proportion of the female face, and so on) (Barber, 1995; Jones, 1995; Wehr, MacDonald, Lindner, \& Yeung, 2001).

\section{Competing Explanations of the Preference for Small Female Feet}

Human foot size is sexually dimorphic - both in absolute terms and, more significantly, relative to stature, women generally have smaller feet than men (Fessler, Haley, \& Lal, in press). Consistent with the above 
discussion, this fact plays a central role in two competing explanations of the postulated positive contribution of small foot size to female attractiveness. First, as proposed above with regard to skin tone, sexual dimorphism in foot size may simply be the result of natural selection; observers may then notice the sex difference in foot size, and may eventually view it as emblematic of differences between the sexes. Over time, people may come to associate small feet with femininity and large feet with masculinity and may, therefore, view as beautiful women who exhibit the hallmark version of the feminine trait. We call this the observational hypothesis. Second, as in the case of cephalofacial morphology, it is possible that sexual dimorphism in foot size is the product, rather than the source, of preferences for small female feet.

Children's feet are smaller than those of adults; hence, a small adult foot may be a cue of youthfulness (Barber, 1995). In addition, in adult women, foot size increases with both age and parity (Bird, Menz, \& Hyde, 1999; Block, Hess, Timpano, \& Serlo, 1985; Chantelau \& Gede, 2002), patterns that augment the potential indexical value of foot size given men's preference for traits associated with youth and nulliparity (Symons, 2002). Sexual dimorphism in foot size may thus have evolved due to a human male preference for women with small, youthful feet (Barber, 1995). We term the proposition that men possess an innate preference for small female foot size the evolutionary hypothesis.

Preliminary evidence in support of the evolutionary hypothesis derives from the fact that, in contrast to the case of skin color, in which sexual dimorphism is potentially explicable entirely in terms of natural selection, dimorphism in foot size does not conform to the pattern expected based solely on functional considerations. During bipedal locomotion, weight is borne in the foot primarily by the heel and the forward metatarsal region (Lundeen, Lundquist, Cornwall, \& McPoil, 1994). Whereas the heel is approximately plumb with the lower leg, and hence bears weight in a largely static fashion, the forefoot, being located forward of the lower leg, bears weight dynamically, with the plantar flexor muscles exerting a downward force on the foot via the flexor tendons (Kirby, 2000). The longer the foot, the more that this dynamic platform is able to stabilize the forward motion of the body. During pregnancy, women carry a substantial anteriorly located mass. This change moves the body's center of gravity upward and, in the absence of postural compensation, forward (Fries \& Hellebrandt, 1943), thus increasing the force of the forward roll borne by the plantar flexor muscles (Foti, Davids, \& Bagley, 2000). Given the significant fitness costs of accidental falls during pregnancy (Dahmus \& Sibai, 1993; Runnebaum,
Holcberg, \& Katz, 1998; Williams, McClain, Rosemurgy, \& Colorado, 1990), one might, therefore, expect natural selection to have favored larger foot size relative to stature in women compared to men. That the opposite pattern occurs suggests that forces other than simple natural selection have shaped human female foot size.

To date, no investigation has systematically explored the possibility that small foot size contributes to female attractiveness in a variety of cultures. We therefore sought to both (1) examine the prevalence of the preference for small female feet across diverse cultures, and (2) pit the observational explanation of such a preference against the evolutionary explanation.

The observational hypothesis predicts that aesthetic preferences should symmetrically polarize the female/male dichotomy. If preferences simply reflect existing morphological differences, then, to the extent that proportionately small feet are equated with a feminine phenotype, proportionately large feet should be equated with a masculine phenotype; accordingly, if possession of proportionately small feet is the preferred female form, possession of proportionately large feet should correspondingly be the preferred male form. In contrast, the evolutionary hypothesis predicts asymmetrical preferences in this regard. Research on other aspects of physical attractiveness has shown that averageness contributes to attractiveness; this pattern may reflect either a generalized preference for prototypicality, an adaptive sensitivity to locally-optimal configurations shaped by stabilizing selection (the "central tendency" hypothesis), or a preference for cues of heterozygosity (Rhodes, Jeffery, Watson, Clifford, \& Nakayama, 2003; Symons, 1979; Thornhill \& Gangestad, 1993). Because the evolutionary explanation argues that the only preference that should obtain for male targets is the default one, this hypothesis predicts that, whereas the ideal female should have proportionately smaller-than-average feet, the ideal male should possess feet of average size proportionate to stature.

\section{METHOD}

\section{Participants}

Targeting geographically and culturally disparate peoples, we selected societies to be investigated on a convenience basis. After obtaining approval from the respective Institutional Review Boards and/or relevant local authorities, we collected data from volunteer participants in urban locations in Iran, India, Russia, Lithuania, Brazil, the U.S., and Cambodia, and in rural locations in Tanzania and Papua New Guinea. Participation rates were 
Table I. Tests of Foot Size Preference in Each of the Nine Countries

\begin{tabular}{lrcc}
\hline Country & $N$ & $\begin{array}{c}\text { Foot size of most } \\
\text { minus least } \\
\text { attractive woman }\end{array}$ & $\begin{array}{c}\text { Extremity of foot } \\
\text { size of most minus } \\
\text { least attractive man }\end{array}$ \\
\hline Iran & 36 & $-2.28^{* *}$ & 0.36 \\
India & 47 & -0.23 & 0.28 \\
Tanzania & 29 & $1.30^{*}$ & 0.22 \\
Lithuania & 102 & $-0.76^{* *}$ & $0.69^{* *}$ \\
Brazil & 48 & $-0.69^{*}$ & $0.56^{* *}$ \\
USA & 150 & $-0.62^{* *}$ & $0.34^{* *}$ \\
PNG & 32 & 0.48 & -0.03 \\
Cambodia & 50 & 0.18 & 0.02 \\
\hline
\end{tabular}

Note. The Russian data are only included in analyses involving just the most attractive drawing.

${ }^{*}$ Differs from 0 at $p<.05$.

${ }^{* *}$ Differs from 0 at $p<.005$.

not recorded, and participants were not paid. ${ }^{12}$ Specifics of the manner in which participation was invited varied by location, ranging from simply continuing an ongoing collaborative ethnographic venture (e.g., Papua New Guinea) to approaching strangers in a subway station (e.g., Russia). Additional information regarding the particulars of each sample is provided in the Appendix. In each case, investigators were fluent in the local language and familiar with local standards for comportment. Although sample sizes varied across cultures (see Table I), efforts were made to recruit approximately equal numbers of women and men in each sample.

\section{Materials}

In order to collect comparable data across disparate cultures, we designed a test that could be easily administered in a wide variety of settings. Although debates, such as those surrounding the work of Singh (1993), suggest that drawings are an imperfect stimulus format for the measurement of aesthetic preferences in the domain of physical attractiveness, they nevertheless have the advantage that they allow for the creation of an artificial phenotype that is not easily classified as belonging to any particular group. We therefore asked an artist to create two simple line drawings, one depicting a woman and one depicting a man. The artist was instructed to select facial features, hair type, and limb proportions that could plausibly be ascribed to a wide variety of populations,

\footnotetext{
${ }^{12}$ In Tanzania and Papua New Guinea, investigators had longstanding relationships with members of local communities; these relationships included the provision of community-wide assistance, aid to key informants, and so on.
}

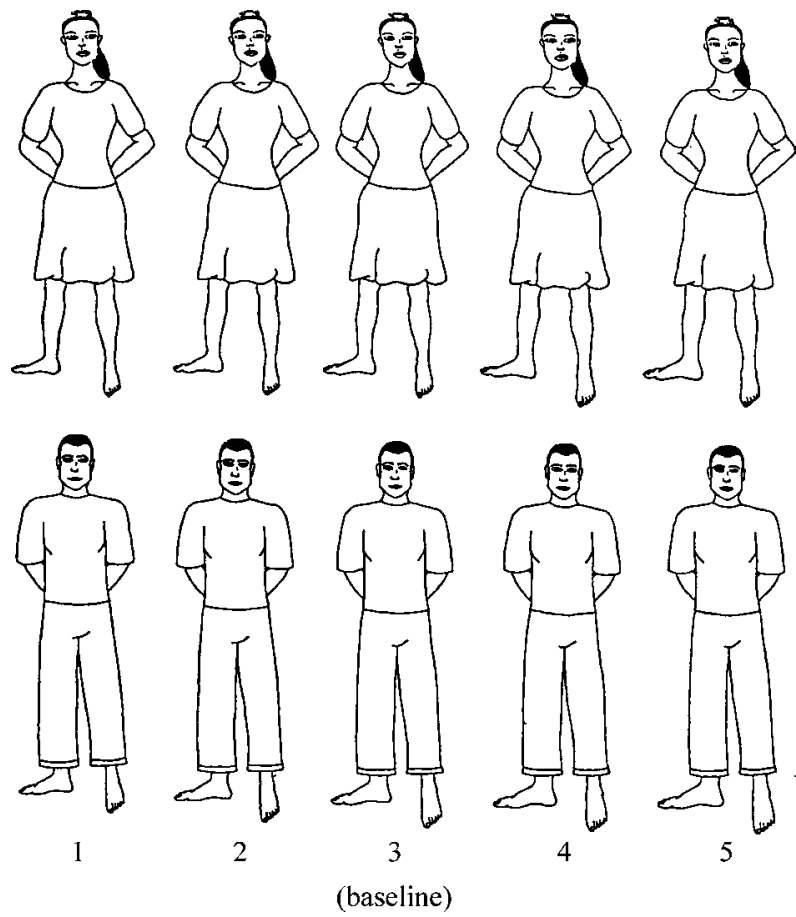

Fig. 1. Stimuli (actual size of each figure $=26 \times 10 \mathrm{~cm}$ ).

and to dress the figures in relatively generic clothing. To ensure that the principal proportions of the foot would be easily visible, the figures were posed with one foot facing directly toward the viewer and one foot perpendicular to the viewer. ${ }^{13}$ The artist was given license to draw the figures as she liked, but was instructed that, consistent with patterns documented in a variety of populations (reviewed in Fessler, Haley, \& Lal, in press), (1) the female figure should have a slightly smaller foot proportionate to stature than the male figure, and (2) foot length should be in the neighborhood of $15 \%$ of stature. The resulting baseline female and male drawings, which we termed drawing \#3, are presented in miniature in Fig. 1. Using a Lanier 5265 digital photocopier, we made four duplicates of each baseline drawing, then removed the feet from each of these duplicates. Next, we employed the zoom function on the photocopier to enlarge the baseline drawing by $10 \%$. Tracing the feet from the enlarged copy onto one of the duplicates and adjusting features of the upper foot and ankle in order to produce a natural appearance, we created the first above-baseline drawing, termed drawing \#4 (see Fig. 1). This drawing was then itself enlarged by $10 \%$ and the feet were traced onto another duplicate of

\footnotetext{
${ }^{13}$ The figures were also posed with their hands behind their backs because a separate investigation, not reported here, concerned the role of hand morphology in judgments of attractiveness.
} 
the original figure, creating the second above-baseline drawing, termed drawing \#5 (see Fig. 1). Using the opposite function on the photocopier, we then shrank the feet on the baseline drawing by $10 \%$, creating the first below-baseline drawing, termed drawing \#2 (see Fig. 1), then repeated the process for the second below-baseline drawing, termed drawing \#1 (see Fig. 1). This produced five female figures and five male figures.

Pilot testing at the University of California, Los Angeles indicated that, even though these stimuli presented a substantial range of variation in foot size, the variation was sufficiently subtle that it was not immediately apparent how the drawings differed from one anotherwhen all five pictures were presented simultaneously, pilot participants frequently spontaneously stated that the pictures were identical. We were, therefore, satisfied that participants' responses would likely reflect genuine assessments of the attractiveness of each of the drawings, and would be relatively free of demand characteristics, as it was not evident to pilot participants that our investigation focused on the role of foot size in attractiveness.

Each drawing was laminated in plastic for durability, and a hook-and-loop fastener was glued to the back. Five matching hook-and-loop fasteners were glued to a rigid mounting board, allowing one set of drawings to be presented simultaneously as a horizontal array. The identification number for each figure was written on the back of the laminated drawing in order to make it easier for the investigator to properly configure the array for each participant (see below); these numbers were not visible when the prepared array was viewed by the participant.

\section{Procedure}

Each investigator employed an identical set of materials consisting of the stimuli, a mounting board, and a set of random numbers. Participants viewed the array at a distance of approximately $1 \mathrm{~m}$; those waiting to participate were prevented from seeing the array or witnessing the current participant's responses. All participants viewed both the female and the male drawings. The left-to-right sequence of each set of drawings was randomized for each presentation using the random number set (e.g., one participant viewed a female array consisting of, from left to right, drawings $[3,5,4,2,1]$, and a male array consisting of drawings [5, 3, 2, 1, 4]; another participant viewed a female array consisting of drawings $[4,1,5$, $3,2]$, etc.). The female drawings were presented first. Participants were asked to point to the drawing of the woman that they considered the most "sexually attractive" or "beautiful" (these terms were translated independently by each investigator). They were then asked to point to the drawing of the woman that they considered the least "sexually attractive" or "beautiful." In order to collect data for a separate study, the same task was then performed using two additional sets of drawings that did not involve feet (one set depicted a woman with her hands visible and feet hidden, the other set portrayed disembodied hands). The male drawings were then presented accompanied by the same instructions, with appropriate changes in vocabulary ("handsome" rather than "beautiful," etc.). If participants protested that all of the drawings in an array looked alike, the researcher instructed them to "Just choose whichever woman/man you think is most/least attractive." The protocol was identical in each culture with the exception that, due to a misunderstanding, researchers in Russia did not ask participants to indicate the least attractive figure in each array. The Russian data are therefore only included in analyses involving just the most attractive drawing.

\section{Measures}

There were several possible strategies for analyzing the data. One null hypothesis is that the raters' preferences would simply be random with respect to foot size. If this were the case, then the frequency of choice of each of the five drawings would follow a uniform distribution. Our first statistical test in each case was, therefore, a onesample chi-square test to establish whether the pattern of preferences recorded departed significantly from chance. However, while useful, this test was not by itself sufficient for the task at hand, as it would not identify the direction of any departure from randomness, and our hypotheses were specific with regard to direction. Further analyses were, therefore, performed using as the dependent variable the foot size of the drawings chosen (scored from 1 [the drawing with the smallest feet] to 5 [the drawing with the largest feet]). If foot size was unrelated to the attractiveness of a drawing, or if the medium foot size was preferred, then the mean foot size of the most attractive drawing would be 3 . In contrast, both of the experimental hypotheses predicted that, for the female stimuli, the mean foot size of the most attractive drawing would be significantly less than 3 , and that of the least attractive drawing would be significantly greater than 3. For the male stimuli, the observational hypothesis predicted that the foot size of the most attractive drawing would be significantly greater than 3 , and that of the least attractive drawing significantly less than 3. In contrast, the evolutionary hypothesis predicted that, for the male stimuli, the medium (baseline) foot size would 
be preferred compared to both extremes. T-tests were used to test these predictions against the data, both in the pooled data set and in the nine cultures individually. Differences between cultures were investigated by treating the foot size of the drawing chosen (again scored from 1 to 5) as the dependent variable, and carrying out analyses of variance with culture as the independent variable.

\section{RESULTS}

The sizes and composition of the nine samples are provided in the Appendix. The mean age of participants differed significantly between samples $(F[8,528]=$ $5.86, p<.001)$. However, there was no evidence that age of participant affected preferences. In an analysis of variance, the mean age of the participants who chose a given female drawing as most attractive did not differ significantly from the mean age of each of the four sets of participants who selected the other four drawings $(F[4,526]<1)$. The same was true with regard to the mean age of participants who selected a given female drawing as least attractive $(F[4,483]<1)$, the mean age of participants who selected a given male drawing as most attractive $(F[4,520]=1.80, \mathrm{~ns})$, and the mean age of participants who selected a given male drawing as least attractive $(F[4,478]<1)$.

Table II shows the mean foot size of the female and male drawings rated most attractive, and the mean foot size of the female and male drawings rated least attractive, both with participants divided by sex and pooled. As is evident in the table, regardless of the sex of the rater, the mean foot size of the female drawing deemed most attractive was below baseline (smaller than 3 ), and the mean foot size of the female drawing deemed least attractive was above baseline (larger than 3); for the male drawings, both the most and the least attractive drawings had mean foot sizes close to 3. Statistical tests of the significance of these deviations are presented below.

Table II. Mean Foot Size of the Female and Male Drawings Rated Most and Least Attractive, by Sex of Participant

\begin{tabular}{|c|c|c|c|c|c|c|}
\hline & \multicolumn{6}{|c|}{ Participants } \\
\hline & \multicolumn{2}{|c|}{ Male } & \multicolumn{2}{|c|}{ Female } & \multicolumn{2}{|c|}{ Combined } \\
\hline & M & $S D$ & M & $S D$ & M & $S D$ \\
\hline Most attractive female & 2.85 & 1.43 & 2.88 & 1.44 & 2.86 & 1.45 \\
\hline Least attractive female & 3.31 & 1.46 & 3.44 & 1.41 & 3.38 & 1.44 \\
\hline Most attractive male & 3.14 & 1.32 & 2.81 & 1.31 & 2.98 & 1.33 \\
\hline Least attractive male & 2.98 & 1.59 & 3.10 & 1.60 & 3.04 & 1.60 \\
\hline
\end{tabular}

Note. Absolute range, 1.00-5.00.

\section{Female Stimuli}

When the data from the nine samples were pooled, the patterning of the frequency with which each of the female drawings was selected as most attractive differed significantly from a uniform (i.e., random) distribution $\left(\chi^{2}=12.15, d f=4, p<.05\right)$; the same was true of the patterning of the frequency with which each of the female drawings was selected as least attractive $\left(\chi^{2}=48.62\right.$, $d f=4, p<.01$ ).

In the pooled data set, the mean foot size of the drawing rated most attractive was $2.86(S D, 1.45)$, which was significantly less than $3(t[530]=2.19, p<.05)$. The mean size for the least attractive drawing was $3.38(S D, 1.44)$, which was significantly greater than $3(t[487]=5.63, p<.001)$. The drawing chosen most frequently as the most attractive woman was number 1 (the smallest feet), though the effect was not large (Fig. 2, top left panel). The drawing chosen most frequently as the least attractive woman was number 5 , the drawing with the largest feet (Fig. 2, top right panel). A participant's most attractive drawing had smaller feet than his or her least attractive drawing in $59.8 \%$ of cases, and the foot size of the most attractive minus the foot size of the least attractive differed significantly from zero $(M=-.5$, $t[486]=4.57, p<.001)$. Male and female participants did not differ significantly with respect to the foot size of their most attractive $(t[529]<1)$ or least attractive choices $(t[486]<1)$.

The foot size of the female drawing rated most attractive varied significantly across the nine cultures $(F[8,522]=3.18, p=.02)$, as did the foot size of the least attractive female drawing $(F[7,480]=5.79$, $p<.001)$. To investigate this variation, we calculated the foot size of the most attractive minus that of the least attractive drawing for each participant, and tested for the departure of this score from 0 in the nine cultures separately (Table I). As the table shows, the results were significant (most attractive having smaller feet than least attractive) in Iran, Lithuania, Brazil, and the U.S. The trend was in the same direction but not significant in India, whereas in Cambodia and Papua New Guinea there were nonsignificant trends in the opposite direction. Tanzania was the only culture to show a significant contrary trend, with large feet preferred to small.

\section{Male Stimuli}

When the data from the nine samples were pooled, the patterning of the frequency with which each of the 

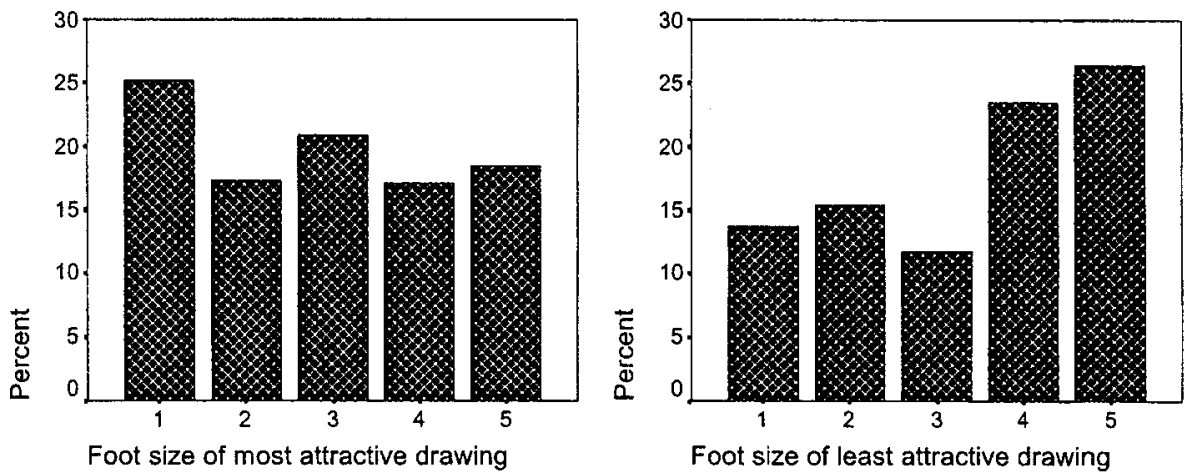

Female stimuli
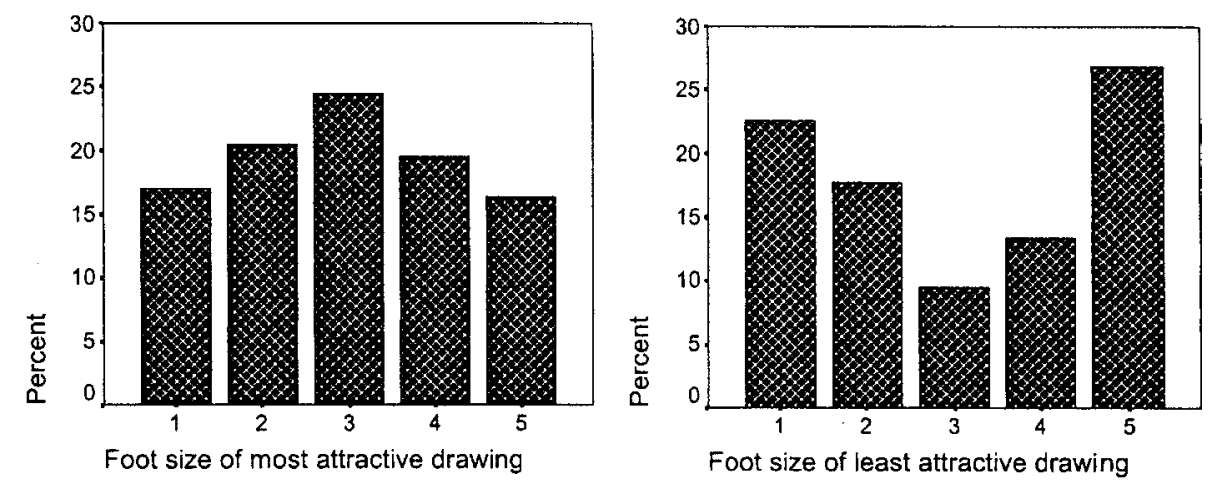

Male stimuli

Fig. 2. Percentages of participants choosing each of the five foot sizes $(1=$ smallest $)$ for the most attractive female stimulus (top left), least attractive female stimulus (top right), most attractive male stimulus (bottom left), and least attractive male stimulus (bottom right).

male drawings was selected as most attractive differed significantly from a uniform distribution $\left(\chi^{2}=11.29\right.$, $d f=4, p<.05)$; the same was true of the patterning of the frequency with which each of the male drawings was selected as least attractive $\left(\chi^{2}=57.24, d f=4\right.$, $p<.01)$.

Inspection of the preference data suggested that the baseline male drawing (drawing \#3) was favored, and both extremes disfavored (Fig. 2, bottom panels). To test this impression statistically, the choices were re-coded according to their degree of extremity. Thus, a choice of stimulus 3 would have an extremity score of 0 , stimulus 2 or 4 would have an extremity score of 1 , and stimulus 1 or 5 would have an extremity score of 2 . The mean extremity score for the most attractive male drawing was 1.07 ( $S D$, 0.77 ), whereas the mean extremity score for the least attractive male drawing was $1.44(S D, 0.68)$. For $51.1 \%$ of raters, the most attractive drawing was less extreme than the least, and for another $22.9 \%$ they were equally extreme. For only $26.0 \%$ was the most attractive more extreme than the least. Individual participants' extremity scores for the most attractive drawing were significantly lower than for the least attractive drawing (paired $t$-test, $t[481]=7.25, p<.001)$. This effect appeared equally in male and female participants (paired $t$-tests, male participants: $t(245)=4.65, p<.001$; female participants: $t(236)=5.64, p<.001)$. Hence, in the pooled data set, the baseline male drawing was indeed favored, while both positive and negative deviations from the baseline male drawing were disfavored.

The difference in extremity between the foot size of the least attractive male drawing and that of the most attractive male drawing differed significantly across cultures $(F[7,474]=2.82, p<.01)$. The mean difference between the extremity of foot size of the most and least attractive male drawing is shown for the cultures separately in Table I. The difference was significant (most attractive drawing less extreme than least attractive) in Lithuania, Brazil, and the U.S., with nonsignificant trends in the same direction in Iran, India, and Tanzania. Papua New Guinea, and Cambodia showed no trend in either direction. 


\section{DISCUSSION}

Our investigation of the role of foot size in judgments of attractiveness indicated that proportionately small feet contributed to female attractiveness, while males having an average foot size relative to stature were generally considered most attractive. These results were significant in the data set overall, and also in several geographically disparate cultures considered individually. However, the effects did not reach statistical significance in all of the cultures. This may be partly due to the small sample sizes, and hence low statistical power, when some of the cultures were considered separately. Notably, high levels of significance were found in Lithuania and the U.S., the cultures with the largest sample sizes. In only one case (Tanzania, for the female stimuli) was there a significant finding in the reverse direction to the predictions.

The female pattern was more evident in regard to disfavored foot size than in regard to favored foot size suggesting that, at least within the context of our procedure, there was greater consensus and/or stronger sentiment regarding what makes a woman unattractive than there was regarding what makes her attractive.

In contrast to the asymmetry between the strength of the patterns in the favored versus disfavored foot size in judgments of female attractiveness, assessments of the most and least attractive male figures were direct inverses of one another (compare the two lower panels of Fig. 2)the baseline (average) figure was chosen most often as the favored, and least often as the disfavored, with steady reciprocal changes in each of these assessments as foot size both increased and decreased. While the contrast between the regularity of the patterns in assessments of female and male attractiveness may have reflected greater heterogeneity in participants' preferences concerning female attractiveness or may have been due to negativity bias (Rozin \& Royzman, 2001) in the latter, it is also possible that this difference simply reflected a practice effect-the female assessment task was the first in a sequence of four such tasks, while the male assessment task was the last in this sequence; hence, participants may have been more comfortable with the task by the time they viewed the male stimuli.

Our findings that small foot size relative to stature often increased female attractiveness while average foot size relative to stature was generally preferable for males are consistent with the hypothesis that humans possess an evolved preference for small female feet as one facet of the general preference for signs of youth and nulliparity in females. The competing explanation, that preferences merely reflect observable differences in relative foot size between men and women, incorrectly predicted that males with large feet should be preferred.
Although the patterns predicted by the evolutionary hypothesis emerged in our cross-cultural data, significant inter-cultural variation was also evident. The most dramatic divergence from the prevailing patterns occurred in Tanzania. We suspect that the Tanzanian findings reflect methodological difficulties. Many Tanzanian participants were confused by the task, with approximately $25 \%$ objecting that it was impossible to select best/worst figures because all the drawings were the same. Less dramatic divergence from the prevailing pattern occurred in Papua New Guinea and Cambodia. At present, we are unable to explain these cases, although similar problems of lack of familiarity with the task context may have constituted methodological confounds (particularly in the Papua New Guinea case), as these three populations have the lowest levels of formal schooling of the nine groups studied (see Appendix). In addition, in at least two of these three cases, definitive conclusions are difficult given small sample sizes. In contrast to these cases, the Iranian results were distinguished by the strength of the effect for the female stimuli in the direction predicted by the sexual selection hypothesis. This may be an example of the cultural elaboration of what is elsewhere a subtle tendency.

While we sought to conduct investigations in diverse cultures, expedience was a principal determinant of the locations selected, and we do not claim to have captured even a fraction of the cultural variation present in the world today. Accordingly, the conclusions presented herein should be considered preliminary. The Tanzanian and Papua New Guinean participants, while not wholly isolated from modern mass media, nonetheless had less contact with it than did the members of the other samples. Given that these two samples failed to show the pattern found in the study overall, we cannot rule out the possibility that the prevailing pattern of preferences reflects cultural diffusion rather than evolved psychology. However, there is reason to doubt this explanation given that the U.S. and Brazil, though similar in regard to foot preferences, differ in regard to many other constituents of female attractiveness (Jones, 1996) despite having roughly equivalent levels of exposure to global media.

Given that we employed simplified drawings as stimuli, caution is in order in interpreting our results. First, because the drawings were not highly realistic, it is possible that they evoked different assessments than those that are employed in evaluating actual human beings. Second, because the size of the ankle was modified in order to accommodate the enlarged or reduced images of feet, it is possible that participants' assessments actually reflect the role of ankle size, rather than foot size, in judgments of attractiveness. Finally, although we attempted to endow the drawings with relatively generic features, hairstyles, and clothing, the populations sampled 
differed in the extent to which the drawings resembled people encountered on a daily basis. It is, therefore, possible that some aspects of our findings (plausibly including the Tanzanian and Papua New Guinean results) reflect the alien nature of the figures portrayed in the drawings.

In contrast to empirically unsubstantiated symbolic and psychodynamic accounts of the role of foot size in attractiveness (e.g., Rossi, 1976; Zerbe, 1985), our cross-cultural investigation provides preliminary evidence that humans possess an evolved predisposition to view proportionately small feet as an attractive feature in women. This pattern is consistent with the general human male predilection for signs of youth and nulliparity in females, and supports the contention that intersexual selection is responsible for the otherwise puzzling sexual dimorphism in human foot size proportionate to stature.

\section{APPENDIX: RESEARCH CONTEXTS}

Initials of respective investigator(s) are presented in parentheses

- Iran (YA): 19 men, 17 women, age 18-83 ( $M=$ $40.25, S D=17.84$ ). Data were collected in the city of Tehran at family gatherings, workplaces, women's showrooms, and other semi-private locations in upper-middle to upper class areas of the city, with a correspondingly relatively high expected level of education. Though mass media are subject to government regulation and censorship, many people in these social classes have private access to Western entertainment.

- India (JM): 26 men, 21 women, age 18-95 $(M=28.04, S D=12.55)$. Data were collected from Hindi speakers in public areas in the city of Varanasi, including a temple and a university hostel. Level of education varied from none to university graduates. Though the region is not cosmopolitan by Indian standards, most people have ready access to television, movies, and print media.

- Russia (AK and DK): 20 men, 23 women, age 18-70 $(M=30.23, S D=14.69)$. Data were collected in public locations throughout Moscow. Approximately one-third of the participants were university students, one-third were universityeducated members of the middle class, and onethird were individuals of varying backgrounds recruited in subway stations. Most residents of Moscow have considerable access to television, movies, and print media.
- Tanzania (MBM): 19 men, 10 women, age 20$48(M=28.86, S D=7.42)$. Data were collected in a village setting among the Pimbwe, an indigenous Bantu population of the Rukwa Valley. The majority of adults have attended some primary school. No print media are available in the village. Entrepreneurs bring a VCR on the back of a bicycle once a month on which they show Indian and Western videos.

- Lithuania (AZ and BG): 42 men, 60 women, age 18-64 ( $M=28.16, S D=10.32)$. Data were collected in homes, offices, and university classrooms in the city of Vilnius. Approximately half of the participants were university students, with the remainder having some post-secondary education. Nearly all of the participants were middle class, with considerable access to print and electronic media.

- Brazil (MC, MOC, IAP, and LSS): 25 men, 23 women, age 18-73 $(M=30.12, S D=13.11)$. Data were collected in public areas such as parks and street corners, university classrooms, and offices in the city of Salvador. The majority of participants were middle class; most had completed high school, and some had postsecondary educations. Most participants likely had considerable access to mass media.

- USA (DF): 77 men, 73 women, age 18-85 ( $M=$ 33.07, $S D=15.70)$. Data were collected in a wide variety of public areas throughout southern California; locations selected were such that most participants were likely members of the uppermiddle and middle classes, with correspondingly high rates of post-secondary education. Access to print and electronic media is extensive in this region.

- Papua New Guinea (AB): 20 men, 12 women, age 16-70 $(M=39.78, S D=16.62)$. Data were collected in village settings among Sursurunga speakers of southern New Ireland Province. The average level of education is six years of primary school. Although newspapers are common, contact with magazines and generator-operated VCRs occurs less than once per year; there are no televisions in the region.

- Cambodia (TD): 25 men, 25 women, age 11-55 $(M=26.02, S D=11.43)$. Data were collected in public locations in the city of Phnom Penh, including an amusement park and a restaurant. These areas are frequented by members of the lower-middle class, individuals who generally have a high school education. Although Western movies and magazine images are available, they 
are largely beyond the reach of most participants; participants generally have access to Cambodian television, which features Western programs only to a limited degree.

\section{ACKNOWLEDGMENTS}

This project was supported by a grant to the first author from the UCLA Council On Research; the second author participated while a visiting faculty fellow at the UCLA Center for Behavior, Evolution, and Culture. We thank Alexander P. Arguello and the members of the UCLA Experimental Biological Anthropology lab for helpful suggestions, and the Editor and three anonymous reviewers for constructive feedback. We are grateful to the many research assistants who aided in this project, particularly Leslie Musser, Anna Heilig, Jeannette Mekdara, and Kristin Lenihan.

\section{REFERENCES}

Barber, N. (1995). The evolutionary psychology of physical attractiveness: Sexual selection and human morphology. Ethology \& Sociobiology, 16, 395-424.

Bird, A. R., Menz, H. B., \& Hyde, C. C. (1999). The effect of pregnancy on footprint parameters. A prospective investigation. Journal of the American Podiatric Medical Association, 89, 405-409.

Block, R. A., Hess, L. A., Timpano, E. V., \& Serlo, C. (1985). Physiologic changes in the foot during pregnancy. Journal of the American Podiatric Medical Association, 75, 297-299.

Buss, D. M. (1994). The evolution of desire: Strategies of human mating. New York: Basic Books.

Chantelau, E., \& Gede, A. (2002). Foot dimensions of elderly people with and without diabetes mellitus: A data basis for shoe design. Gerontology, 48, 241-244.

Curtis, V., Aunger, R., \& Rabie, T. (2004). Quantitative evidence that disgust evolved to protect from risk of disease. Proceedings of the Royal Society: Biology Letters, 272, S131-133.

Dahmus, M. A., \& Sibai, B. M. (1993). Blunt abdominal trauma: Are there any predictive factors for abruptio placentae or maternalfetal distress? American Journal of Obstetrics and Gynecology, $169,1054-1059$.

Dixson, A. F., Halliwell, G., East, R., Wignarajah, P., \& Anderson, M. J. (2003). Masculine somatotype and hirsuteness as determinants of sexual attractiveness to women. Archives of Sexual Behavior, 32, 29-39.

Fessler, D. M. T., Haley, K. J., \& Lal, R. (in press). Sexual dimorphism in foot length proportionate to stature. Annals of Human Biology.

Fink, B., Grammer, K., \& Thornhill, R. (2001). Human (Homo sapiens) facial attractiveness in relation to skin texture and color. Journal of Comparative Psychology, 115, 92-99.

Foti, T., Davids, J. R., \& Bagley, A. (2000). A biomechanical analysis of gait during pregnancy. Journal of Bone and Joint Surgery, 82, 625-632.

Frederick, D. A., \& Haselton, M. G. (2004). Male muscularity as a good genes indicator: Evidence from women's preferences for short-term and long-term mates. Paper presented at the Society for Personality and Social Psychology Conference, Austin, TX, January 31st, 2004.

Frey, C., Thompson, F., Smith, J., Sanders, M., \& Horstman, H. (1993). American Orthopaedic Foot and Ankle Society women's shoe survey. Foot \& Ankle, 14, 78-81.
Fries, E. C., \& Hellebrandt, F. A. (1943). The influence of pregnancy on the location of the center of gravity, postural stability, and body alignment. American Journal of Obstetrics and Gynecology, 46, 374-380.

Grammer, K., \& Thornhill, R. (1994). Human (Homo sapiens) facial attractiveness and sexual selection: The role of symmetry and averageness. Journal of Comparative Psychology, 108, 233-242.

Jablonski, N. G., \& Chaplin, G. (2000). The evolution of human skin coloration. Journal of Human Evolution, 39, 57-106.

Jackson, B. (2000). Splendid slippers: A thousand years of an erotic tradition. Berkeley, CA: Ten Speed Press.

Jones, D. M. (1995). Sexual selection, physical attractiveness and facial neoteny: Cross-cultural evidence and implications. Current Anthropology, 36, 723-748.

Jones, D. M. (1996). Physical attractiveness and the theory of sexual selection: Results from five populations. Ann Arbor, MI: University of Michigan Press.

Kaar, P., \& Jokela, J. (1998). Natural selection on age-specific fertilities in human females: Comparison of individual-level fitness measures. Proceedings of the Royal Society of London Series B Biological Sciences, 265, 2415-2420.

Kirby, K. A. (2000). Biomechanics of the normal and abnormal foot. Journal of the American Podiatric Medical Association, 90, 30-34.

Lundeen, S., Lundquist, K., Cornwall, M. W., \& McPoil, T. G. (1994). Plantar pressure during level walking compared with other ambulatory activities. Foot \& Ankle International, 15, 324-328.

Manson, J. H. (1997). Does female rank or age affect mate choice behavior in free-ranging rhesus macaques? Folia Primatologica, $68,366-369$.

Nettle, D. (2002). Height and reproductive success in a cohort of British men. Human Nature, 13, 473-491.

Rhodes, G., Jeffery, L., Watson, T. L., Clifford, C. W. G., \& Nakayama, K. (2003). Fitting the mind to the world: Face adaptation and attractiveness aftereffects. Psychological Science, 14, 558.

Rossi, W. A. (1976). The sex life of the foot and shoe. New York: Saturday Review Press.

Rozin, P., \& Royzman, E. B. (2001). Negativity bias, negativity dominance, and contagion. Personality \& Social Psychology Review, 5 , 296-320.

Runnebaum, I. B., Holcberg, G., \& Katz, M. (1998). Pregnancy outcome after repeated blunt abdominal trauma. European Journal of Obstetrics, Gynecology, and Reproductive Biology, 80, 85-86.

Singh, D. (1993). Body shape and women's attractiveness: The critical role of waist-to-hip ratio. Human Nature, 4, 297-321.

Symons, D. (1979). The evolution of human sexuality. New York: Oxford University Press.

Symons, D. (1995). Beauty is in the adaptations of the beholder: The evolutionary psychology of human female sexual attractiveness. In P. R. Abramson \& S. D. Pinkerton (Eds.), Sexual nature/sexual culture (pp. 80-118). Chicago: University of Chicago Press.

Symons, D. (2002, January). Warrior lovers: Erotic fiction, evolution, and female sexuality. Paper presented at the UCLA/UCSB Evolution, Mind and Behavior Conference, Santa Barbara, CA.

Thornhill, R., \& Gangestad, S. W. (1993). Human facial beauty: Averageness, symmetry, and parasite resistance. Human Nature, $4,237-269$.

Van den Berge, P., \& Frost, P. (1986). Skin color Preference, sexual dimorphism, and sexual selection: A case of gene culture coevolution? Ethnic and Racial Studies, 9, 87-113.

Wehr, P., MacDonald, K., Lindner, R., \& Yeung, G. (2001). Stabilizing and directional selection on facial paedomorphosis: Averageness or juvenilization. Human Nature, 12, 383-402.

Williams, J. K., McClain, L., Rosemurgy, A. S., \& Colorado, N. M. (1990). Evaluation of blunt abdominal trauma in the third trimester of pregnancy: Maternal and fetal considerations. Obstetrics and Gynecology, 75, 33-37.

Zerbe, K. J. (1985). "Your feet's too big": An inquiry into psychological and symbolic meanings of the foot. Psychoanalytic Review, 72, 301-314. 